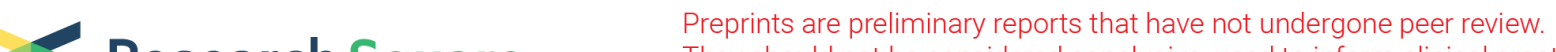 Research Square
or referenced by the media as validated information.
}

\section{Reduced Mortality From KPC-K.Pneumoniae Bloodstream Infection in High-Risk Patients With Hematological Malignancies Colonized by KPC-K.Pneumoniae}

\author{
Alessandra Micozzi ( $\nabla$ alessandra.micozzi@uniroma1.it ) \\ Universita degli Studi di Roma La Sapienza \\ Giuseppe Gentile \\ Sapienza University of Rome: Universita degli Studi di Roma La Sapienza \\ Stefania Santilli \\ Azienda Policlinico Umberto I: Umberto I Policlinico di Roma
}

Clara Minotti

Azienda Policlinico Umberto I: Umberto I Policlinico di Roma

\section{Saveria Capria}

Azienda Policlinico Umberto I: Umberto I Policlinico di Roma

\section{Maria Luisa Moleti}

Azienda Policlinico Umberto I: Umberto I Policlinico di Roma

\section{Walter Barberi}

Azienda Policlinico Umberto I: Umberto I Policlinico di Roma

\section{Claudio Cartoni}

Azienda Policlinico Umberto I: Umberto I Policlinico di Roma

\section{Silvia Maria Trisolini}

Azienda Policlinico Umberto I: Umberto I Policlinico di Roma

\section{Anna Maria Testi}

Sapienza University of Rome: Universita degli Studi di Roma La Sapienza

\section{Anna Paola lori}

Azienda Policlinico Umberto I: Umberto I Policlinico di Roma

\section{Giampaolo Bucaneve}

Ospedale Santa Maria della Misericordia: Presidio Ospedaliero Universitario Santa Maria della Misericordia

\section{Robin Foa}

Sapienza University of Rome: Universita degli Studi di Roma La Sapienza 
Keywords: KPC-K.pneumoniae, hematological malignanciy, carriers, bacteremia, mortality, initial active treatment

Posted Date: October 1st, 2020

DOI: https://doi.org/10.21203/rs.3.rs-74911/v1

License: (c) (1) This work is licensed under a Creative Commons Attribution 4.0 International License. Read Full License 


\section{Abstract}

Background. KPC-K.pneumoniae bloodstream infection (KPC-KpBSI) mortality rate in patients with hematological malignancies is reported about $60 \%$. The initial treatment active against KPCK.pneumoniae is crucial for survival and KPC-K.pneumoniae rectal colonization usually precedes KPC$\mathrm{KpBSI}$. We evaluated the impact on KPC-KpBSI mortality of the preemptive use of antibiotics active against KPC-K.pneumoniae, as opposed to inactive or standard empiric antibiotics, for the empiric treatment of febrile neutropenia episodes in patients with hematological malignancy identified as KPCK.pneumoniae intestinal carriers.

Methods. We compared the outcomes of KPC-KpBSIs occurring in high-risk patients with hematological malignancy known to be colonized with KPC-K.pneumoniae, during two time periods:

March 2012-December 2013 (Period 1, initial approach to KPC-K.pneumoniae spread) and January 2017October 2018 (Period 2, full application of the preemptive strategy). The relative importance of the various prognostic factors that could influence death rates were assessed by forward stepwise logistic regression models.

Results. KPC-KpBSI-related mortality in patients with hematological malignancies identified as KPCK.pneumoniae carriers dropped from $50 \%$ in Period 1 to $6 \%$ in Period 2 ( $p<0.01$ ), from $58 \%$ to $9 \%$ in acute myeloid leukemia carriers $(\mathrm{p}<0.01)$. KPC-KpBSIs developed in patients identified as KPC-K.pneumoniae carriers were treated with initial active therapy in $56 \%$ and $100 \%$ of cases in Period 1 and Period 2 , respectively ( $p<0.01)$, consisting in active antibiotic combinations in $39 \%$ and $94 \%$ of cases, respectively $(p<0.01$ ). In Period 1, the $61 \%$ of KPC-KpBSI were breakthrough (fatal in the $73 \%$ of cases) while no breakthrough KPC-KpBSI was observed in Period 2 ( $p<0.01)$. Overall, KPC-KpBSI-related mortality was $88 \%$ with no initial active treatment, $11.5 \%$ with at least one initial active antibiotic $(p<0.01), 9 \%$ with initial active combination. Only the initial active treatment resulted independently associated with survival.

Conclusions. In high-risk patients with hematological malignancies colonized by KPC-K.pneumoniae, the empiric treatment of febrile neutropenia active against KPC-K.pneumoniae reduced KPC-KpBSI-related mortality to $6 \%$ and prevented fatal breakthrough KPC-KpBSI.

\section{Introduction}

The mortality rate associated with KPC-K.pneumoniae bloodstream infection (KPC-KpBSI) in neutropenic patients with hematological malignancies is reported up to $60 \%(1-10)$. The initial active antibiotic treatment is crucial for KPC-KpBSI survival (11-12), especially in high-risk vulnerable patients such as acute leukaemia patients undergoing intensive chemotherapy, and hematopoietic stem cell transplant (HSCT) recipients (7-10). A delay in KPC-KpBSI active antibiotic treatment is common in these patients since in most cases the recommended empiric antibiotic regimens for febrile neutropenia contain antibiotics without in vitro activity against carbapenem-resistant Enterobacteriaceae (CRE). Colonization 
is a recognized predictive factor for $\mathrm{KPC}-\mathrm{KpBSI}$ occurrence $(10,13-14)$, and the prevalence of colonized hematological patients hospitalized for chemotherapy treatments is increasing in Italy and many other countries with high KPC-K.pneumoniae nosocomial diffusion (15). On March 2012, KPC-K.pneumoniae spread at the Hematology Department of Sapienza University of Rome (Italy): we reported the $71 \%$ of KPC-KpBSI mortality rate, and a high rate of KPC-K.pneumoniae rectal carriers who developed KPC-KpBSI and died (8). Good-quality studies on CRE-BSI treatment in neutropenic patients were not available at that time, and recommendations were not sufficiently focused (16-17). We implemented the prompt identification of patients hospitalized who were KPC-K.pneumoniae carriers and we progressively increased the preemptive use of antibiotics active against KPC-K.pneumoniae for the empiric treatment of febrile neutropenia episodes in KPC-K.pneumoniae carriers. Options for CRE treatment are limited (19), and data on hematological patients scarce. Several issues pertain the clinical use of colistin, dosing, pharmacokinetics in critically ill patients, nephrotoxicity and uncertainties about susceptibility testing (12), moreover the emergence of resistance decreases the activity of colistin and other available antibiotics (20). Currently, evidences and recommendations support the use of active antibiotic combinations to treat serious CRE infections (12, 21-23). New combinations with $\beta$-lactamase inhibitors, including carbapenem/ $\beta$-lactamase inhibitor combinations, represent therapeutic alternatives (24-26) to limit colistin use and improve CRE treatment. Good response rates are reported with the use of the ceftazidime-avibactam for targeted or salvage treatment of KPC-KpBSI in immunocompetent patients (24-25), while data in the specific setting of patients with hematological malignancies are limited (27).

We evaluated the impact of the preemptive strategy on KPC-KpBSI-related mortality in high-risk hematological KPC-K.pneumoniae carriers comparing the initial approach to KPC-K.pneumoniae spread and the routine application of the preemptive use of active therapy for the empiric treatment of febrile neutropenia.

\section{Patients And Methods}

We compared KPC-KpBSI developed in patients with hematological malignancies identified as KPCK.pneumoniae carriers attending the Hematology Department of Sapienza University of Rome between March 2012- December 2013 (Period 1) and January 2017-October 2018 (Period 2).

\section{Setting}

The Hematology Department consists of 5 wards and 52 beds. The majority of patients are hospitalized for intensive chemotherapy treatments for acute leukemia and/or HSCT.

\section{Screening for KPC-K.pneumoniae colonization}

From March 2012, when KPC-K.pneumoniae spread at the Hematology Department, the screening for KPC-K.pneumoniae rectal colonization (13) was performed weekly in patients hospitalized in the ward where KPC-K.pneumoniae was first isolated. From September 2012, the screening was extended to all patients hospitalized in the Hematology Department, from October 2013, rectal swabs were collected also 
prior to admission. From January 2018, hospitalized acute leukaemia patients were screened twice weekly. KPC-K.pneumoniae colonized patients underwent contact precautions.

\section{Active empiric antibiotic treatments for febrile neutropenia in KPC-K.pneumoniae carriers.}

Combination of colistin ( 9 million international units [IU] loading dose, then 4.5 million UI q12h) with tigecycline (100 mg loading dose, then $50 \mathrm{mg} \mathrm{q12h}$ ) and/or gentamycin ( $3 \mathrm{mg} / \mathrm{kg} /$ die once-a-day), highdose carbapenems (meropenem $2 \mathrm{~g}$ q8h), double carbapenem (meropenem plus ertapenem $1 \mathrm{~g}$ once-aday) and, from September 2017, ceftazidime-avibactam ( $2.5 \mathrm{~g} \mathrm{q} 8 \mathrm{~h}$ ) as monotherapy or combined with tigecycline and/or gentamycin.

\section{Clinical data recorded}

Data collected from Period 1 and Period 2: a) number of KPC-KpBSI occurred in KPC-K.pneumoniae carriers. b) number of hospitalized patients identified as KPC-K.pneumoniae carriers. c) type of hematological malignancy and chemotherapy treatment administered in the colonized patients with KPC$\mathrm{KpBSI}$. d) length of neutropenia ( $<1000$ neutrophils/cmm) and profound neutropenia $(<100$ neutrophils/cmm) episodes. e) clinical presentation of KPC-KpBSI. f) antibiotic treatment. g) KPC-KpBSI outcome.

\section{Microbiological studies}

In Period 1, species identification and MIC determination of K.pneumoniae isolates were performed using the BD Phoenix automated microbiology system (Becton Dickinson Italia S.p.A Milano, Italy). MICs of imipenem, meropenem, ertapenem, gentamicin, colistin and tigecycline were also evaluated using Etest (BioMerieux Italia S.p.A., Firenze, Italy). In Period 2, species identification was performed using MALDITOF and susceptibility testing was determined using the automated VITEK2 system (bioMérieux, Marcyl'Étoile, France). K.pneumonia blood isolates KPC genetic mechanism was determined with in vitro realtime PCR assay Xpert Carba-R assay (Cepheid, Sunnyvale,CA). For the 34 KPC-K.pneumonia blood isolates, MICs for imipenem, meropenem, ertapenem colistin, tigecycline, gentamicin and ceftazidimeavibactam were determined by broth microdilution (Sensititre Gram Negative MIC Plate, ThermoFischer Scientific, USA) and results were interpreted in accordance with the 2019 breakpoints proposed by the European Committee on Antimicrobial Susceptibility Testing(EUCAST 2019) (28).

\section{Statistical analysis}

Continuous variables were compared using the Kruskal-Wallis test. Categorical variables were compared with the Chi-Square test corrected for continuity or the Fisher's exact test when indicated; $95 \%$ confidence intervals (Cls) for the differences of means and proportions were calculated. Forward stepwise logistic regression models were used to assess the relative importance of the various prognostic factors that could influence the occurrence of death in patient populations reported in population (7-8): acute myeloid leukemia (AML), intensive chemotherapy, shock at onset, breakthrough KPC-KpBSI, active initial therapy. 
The statistical calculations were carried out using the SPSS statistical package (SPSS for Windows, Release 15.0).

\section{Results}

Eighteen KPC-KpBSI developed among 27 patients identified as KPC-K.pneumoniae carriers in Period 1 and $16 \mathrm{KPC}-\mathrm{KpBSI}$ among $88 \mathrm{KPC}-\mathrm{K}$.pneumoniae carriers in Period 2 were compared. The rate of KPCK.pneumoniae colonized patients who developed KPC-KpBSI reduced from 67\% (18/27) in Period 1 to $11 \%(16 / 88)$ in Period 2, $\mathrm{p}<0.01$ (Table 1). 
Table 1

Characteristics of patients with hematological malignancies KPC-K.pneumoniae carriers who developed $\mathrm{KPC}-$ K.pneumoniae BSI.

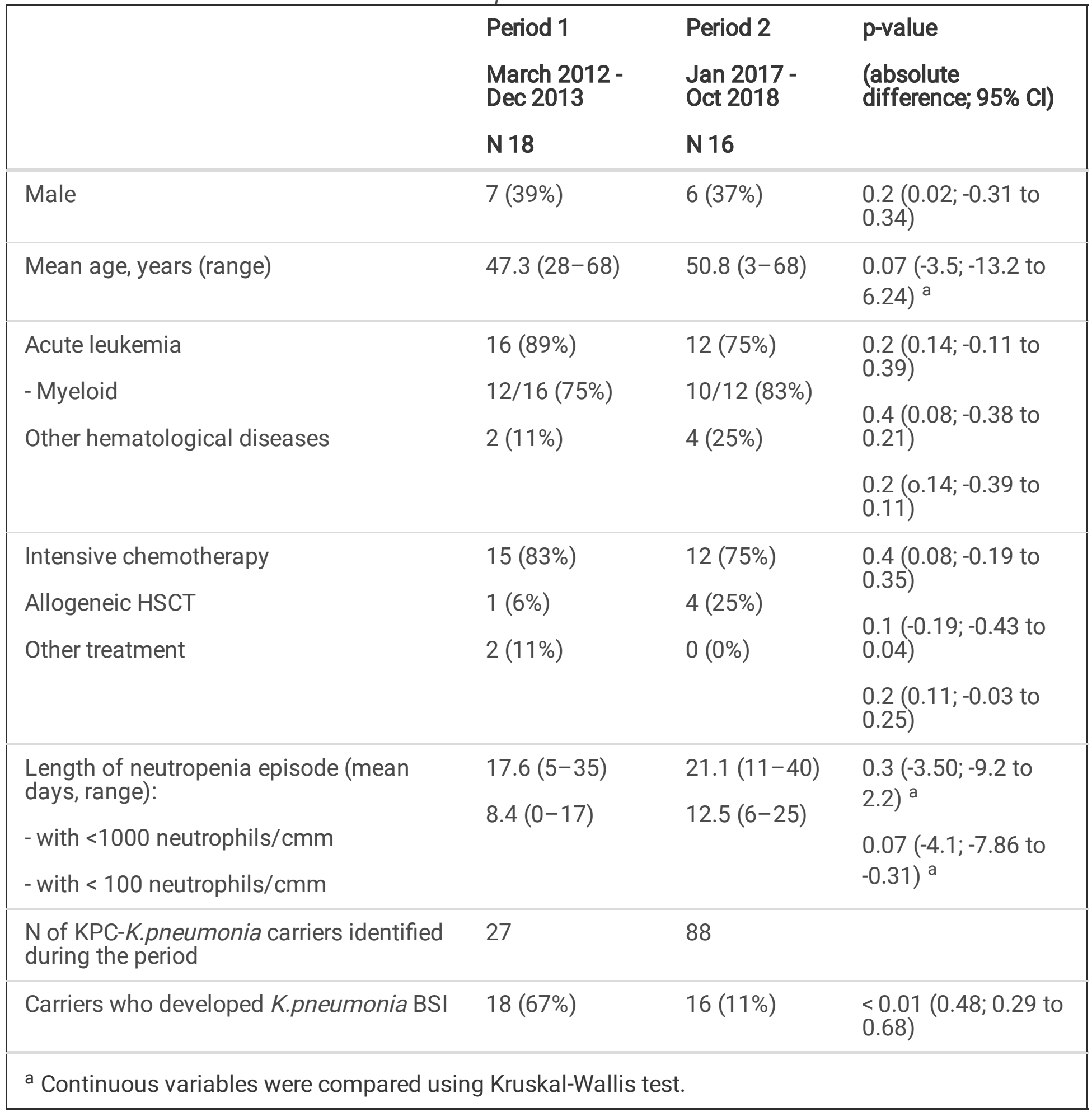

As shown in Table 1, the characteristics of the KPC-K.pneumoniae carriers with KPC-KpBSI were similar in the two periods: the large majority of patients had acute leukaemia, mostly acute myeloid leukaemia (AML) undergoing intensive chemotherapy and they developed comparable neutropenia and profound neutropenia episodes. In both periods, KPC-KpBSI occurred during profound neutropenia in the majority of cases, and presented with shock in about half of the patients (Table 2). The $61 \%(11 / 18)$ of KPC-KpBSI 
occurred in Period 1 were breakthrough, developing in patients who were already receiving inactive systemic antibiotics started (for 4.2 mean days, range 2-8 days) for the empiric treatment of a previous febrile neutropenia episode. None of KPC-KpBSI developed in Period 2 was breakthrough $(p<0.01)$. 
Table 2

Clinical characteristic of KPC-K.pneumoniae BSI.

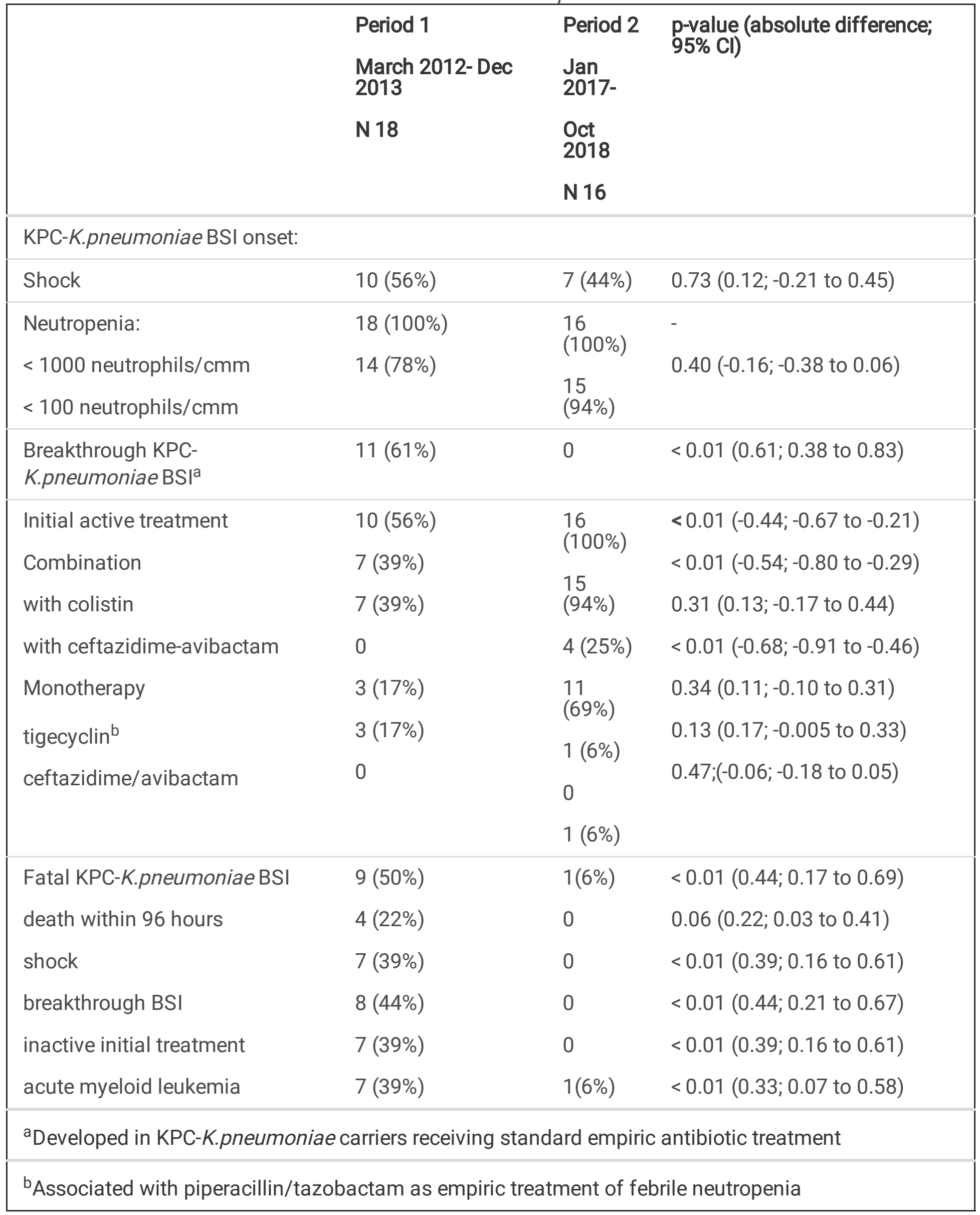


As shown in Table 2, the $56 \%$ of KPC-KpBSI occurred in Period 1 and $100 \%$ of those occurred in Period 2 were treated with active therapy from the very onset, at least one active antibiotic, preemptively administered as empiric treatment of febrile neutropenia $(p<0.01)$. An active combination was used in $39 \%$ and $94 \%$ of cases in Period 1 and Period 2, respectively $(p<0.01)$ (Table 2). In Period 2, 4 of $16(25 \%)$ KPC-KpBSI patients, negative at previous KPC-K.pneumoniae rectal screenings performed during the hospitalization, resulted as new carriers on the same day of febrile neutropenia onset and therefore received active antibiotics as empiric treatment.

KPC-KpBSI-related mortality reduced from $50 \%$ in Period 1 (9/18) to $6 \%$ in Period $2(1 / 16), p<0.01$. Notably, KPC-KpBSI mortality rate in AML carriers decreased from $44 \%(7 / 16)$ in Period 1 to $8 \%(1 / 12)$ in Period 2, $\mathrm{p}<0.01$ (Table 1). The rate of patients who died for KPC-KpBSI among those identified as KPCK.pneumoniae carriers reduced from 33\% (9/27) in Period 1 to $1.1 \%(1 / 88)$ in Period $2, p<0.01$.

In Period 1, fatal KPC-KpBSI presented with shock in $7(78 \%)$ of cases and death occurred within 1 week in 6 patients (66\%), within 96 hours in 4 of them. Fatal KPC-KpBSI were breakthrough in the 89\% (8/9) of cases (the breakthrough KPC-KpBSI mortality rate was 73\%) and in the 78\% (7/9) of cases were initially treated with inactive antibiotics. In Period 2, one AML patient with severe allogeneic-HSCT-related complications, died 10 days after KPC-KpBSI onset.

Overall, KPC-KpBSI observed in Period 1 and Period 2 were fatal in $29 \%(10 / 34)$ of cases: KPC-KpBSI mortality rate was $88 \%$ in patients not receiving initial active antibiotics, $11.5 \%$ in patients initially treated with at least one active antibiotic $(p<0.01)$. Notably, KPC-KpBSI mortality rate was $25 \%$ in patients treated with a single active antibiotic and $9 \%$ in those treated with active combinations. At univariate analysis, initial inactive treatment, breakthrough KPC-KpBSI and KPC-KpBSI occurrence in Period 1, resulted risk factors for mortality (Table 3). In the forward stepwise logistic regression analysis (Table 4), if the initial active therapy was not considered (Model 1), breakthrough KPC-KpBSI mortality rate resulted independently associated with death, but when the initial active therapy was added (Model 2), it resulted the only factor independently associated with survival. In both models, AML as underlying diseases, shock and intensive chemotherapy were not predictors of mortality. 
Table 3

Univariate Analysis of Risk Factors for Mortality

\begin{tabular}{|c|c|c|c|}
\hline & $\begin{array}{l}\text { Survivors } \\
\text { N } 24\end{array}$ & $\begin{array}{l}\text { Non- } \\
\text { survivors } \\
\text { N } 10\end{array}$ & $\begin{array}{l}\text { p-value } \\
\text { (absolute difference; } 95 \% \\
\text { Cl) }\end{array}$ \\
\hline Male & $8(33 \%)$ & $5(50 \%)$ & $0.4(-0.17 ;-0.52$ to 0.19$)$ \\
\hline Mean age, years (range) & $\begin{array}{l}47.7(3- \\
68)\end{array}$ & $52(35-68)$ & $0.6(-4.30 ;-14.9 \text { to } 6.32)^{a}$ \\
\hline Acute leukemia & $19(79 \%)$ & $9(90 \%)$ & $0.41(-0.11 ;-0.35$ to 6.32$)$ \\
\hline - myeloid & $14(58 \%)$ & $8(80 \%)$ & $0.21(-0.22 ;-0.53$ to 0.10$)$ \\
\hline Other hematological disease & $5(21 \%)$ & $1(10 \%)$ & $0.41(-0.21 ;-0.53$ to 0.10$)$ \\
\hline Intensive chemotherapy & $20(83 \%)$ & $8(80 \%)$ & $0.58(0.03 ;-0.25$ to 0.32$)$ \\
\hline Other chemotherapy & $1(4 \%)$ & - & $0.70(0.04 ;-0.03$ to 0.12$)$ \\
\hline Allogeneic stem cell transplant & $3(12 \%)$ & $2(20 \%)$ & $0.46(-0.08 ;-0.35$ to 0.20$)$ \\
\hline $\begin{array}{l}\text { Length of neutropenia episode (mean days, } \\
\text { range) }\end{array}$ & $\begin{array}{l}19.4(5- \\
40)\end{array}$ & $\begin{array}{l}18.9(8- \\
35)^{-}\end{array}$ & $\begin{array}{l}0.70(0.50 ;-5.90 \text { to } 6.90) \\
\text { a }\end{array}$ \\
\hline $\begin{array}{l}\text { with }<1000 \text { neutrophils } / \mathrm{mmc} \\
\text { with }<100 \text { neutrophils } / \mathrm{mmc}\end{array}$ & $\begin{array}{l}11.2(5- \\
25)\end{array}$ & $8.3(0-17)$ & $\begin{array}{l}0.29(2.90 ;-3.29 \text { to } 9.09) \\
\text { a }\end{array}$ \\
\hline Onset of KPC-K.pneumoniae BSI: & $10(42 \%)$ & $7(70 \%)$ & $0.12(-0.28 ;-0.62$ to 0.06$)$ \\
\hline Shock & $21(87 \%)$ & $8(80 \%)$ & $0.61(0.07 ;-0.20$ to 0.35$)$ \\
\hline \multicolumn{4}{|l|}{$<100$ neutrophils/mmc } \\
\hline Breakthrough KPC-K.pneumoniae BSI ${ }^{\mathrm{b}}$ & $3(12.5 \%)$ & $8(80 \%)$ & $\begin{array}{l}<0.01(-0.67 ;-0.95 \text { to } \\
-0.39)\end{array}$ \\
\hline
\end{tabular}

${ }^{a}$ Continuous variables were compared using Kruskal-Wallis test.

${ }^{\mathrm{b}}$ Developed in KPC-K.pneumoniae carriers receiving standard empiric antibiotic treatment

${ }^{\mathrm{C}}$ Associated with piperacillin/tazobactam as empiric treatment of febrile neutropenia (18) 


\begin{tabular}{|llll|}
\hline & $\begin{array}{l}\text { Survivors } \\
\mathbf{N} 24\end{array}$ & $\begin{array}{l}\text { Non- } \\
\text { survivors } \\
\mathbf{N} 10\end{array}$ & $\begin{array}{l}\text { P-value } \\
\text { (absolute difference; } 95 \%\end{array}$ \\
\hline Cl)
\end{tabular}


Table 4

Multivariate Models of risk factors for 30 days crude mortality in patients population (Forward Stepwise logistic regression).

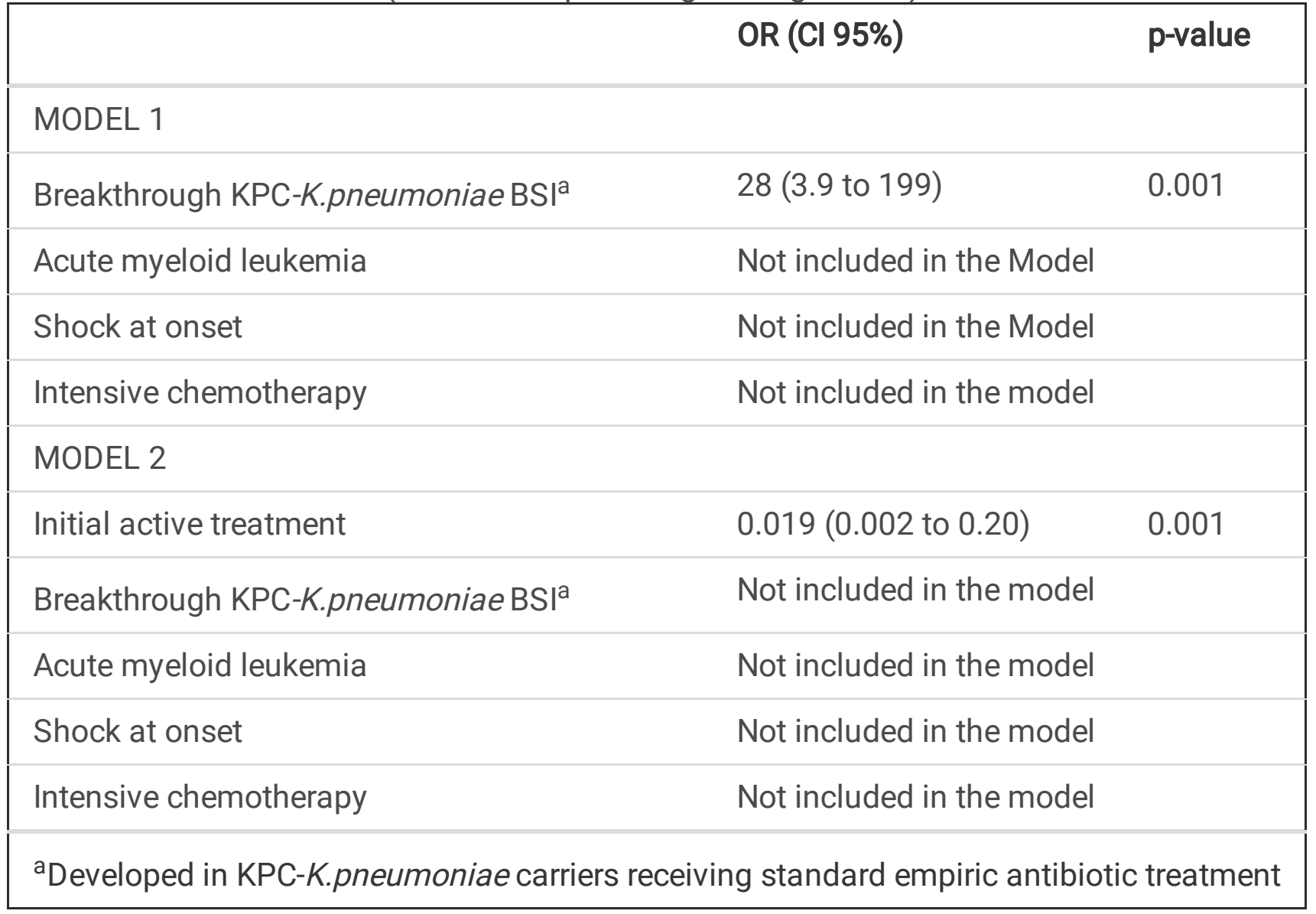

The susceptibilities to antibiotics of the 34 KPC-K.pneumoniae blood isolates are shown in Table 5. According to 2019 EUCAST breakpoints (38), colistin resistant strains were $33 \%$ and $22.5 \%$ in Period 1 and Period 2, respectively. A higher tigecyclin activity was observed in Period 2, all the isolates in Period 1 compared to $50 \%$ in Period 2 had tigecyclin MIC $>0.5 \mathrm{mg} / \mathrm{L}(\mathrm{p}<0.01)$. All isolates resulted susceptible to ceftazidime-avibactam. 
Table 5

Susceptibilty of KPC-K.pneumoniae blood isolates determined by broth microdilution according to 2019 EUCAST breakpoints (38).

\begin{tabular}{|c|c|c|c|c|}
\hline & Period 1 & & Period 2 & \\
\hline \multirow[t]{3}{*}{$\mathrm{N}$ of blood isolates } & 18 & & 16 & \\
\hline & $\begin{array}{l}\text { MIC } \\
\text { range }\end{array}$ & $\begin{array}{l}\mathrm{N}(\%) \text { of susceptible } \\
\text { isolates }\end{array}$ & $\begin{array}{l}\text { MIC } \\
\text { range }\end{array}$ & $\begin{array}{l}\mathrm{N}(\%) \text { of susceptible } \\
\text { isolates }\end{array}$ \\
\hline & $(\mathrm{mg} / \mathrm{L})$ & & $(\mathrm{mg} / \mathrm{L})$ & \\
\hline colistin & $0.25-32$ & $11(67)$ & $0.5-4$ & $14(87.5)$ \\
\hline tigecycline & $2-8$ & 0 & $0.5-2$ & $8(50)$ \\
\hline gentamicin & $\begin{array}{l}1.5- \\
>256\end{array}$ & $8(44)$ & $1->8$ & $8(50)$ \\
\hline $\begin{array}{l}\text { ceftazidime- } \\
\text { avibactam }\end{array}$ & $\begin{array}{l}0.5 / 4- \\
8 / 4\end{array}$ & $18(100)$ & $\begin{array}{l}0.5 / 4- \\
8 / 4\end{array}$ & $16(100)$ \\
\hline meropenem & $>32$ & 0 & $>32$ & 0 \\
\hline imipenem & $>32$ & 0 & $>32$ & 0 \\
\hline ertapenem & $>32$ & 0 & $>32$ & 0 \\
\hline
\end{tabular}

\section{Discussion}

Rectal colonization is a recognized predictive factor for CR-KpBSI occurrence (13-14). Patients with hematological malignancies colonized with CR-K.pneumoniae, mostly AML patients, are at high risk of developing $\mathrm{BSI}(4,8,10)$. In this population, the reported CR-KpBSI mortality rate is dramatic (1-10), mainly in acute leukaemia patients (7-8). AML, intensive chemotherapy and severe and/or prolonged neutropenia were associated with CR-KPBSI-related death (7-8), and septic shock and acute respiratory failure resulted predictors of mortality (7).

This study focuses on KPC-KpBSI-related mortality in high-risk patients with hematological malignancies. The prompt identification of KPC-K.pneumoniae carriers, and the preemptive antibiotic strategy applied to all the carriers -KPC-K.pneumoniae active therapy for the empiric treatment of febrile neutropenia- was associated with the drop of KPC-KpBSI-related mortality from 50\%, observed between March 2012December 2013, to 6\% recorded 3 years later, between January 2017-October 2018. To our knowledge, this is the lowest rate reported so far.

KPC-K.pneumoniae colonized patients who developed BSI in the two periods had comparable high risk factors for KPC-KpBSI-related death: the majority had acute leukaemia, mainly $\mathrm{AML}$, and had received intensive chemotherapy developing profound and prolonged neutropenia, KPC-KpBSI presented with shock in a similar proportion of patients, but KPC-KpBSI mortality rate was significantly higher in Period 
1. Initial treatment with active antibiotics is crucial for survival to CR-KpBSI $(7-8,11-12)$, and the $78 \%$ of patients who died from KPC-KpBSI in Period 1 had received inactive initial antibiotic treatment.

KPC-KpBSI treatment based on culture results presents a high risk of delaying or never receiving appropriate therapy $(1,5)$. In hematological neutropenic KPC-Kpneumoniae carriers at high risk of KPC$\mathrm{KpBSI}(8-10)$, the initial appropriate treatment can be ensured by the preemptive administration of active therapy at any febrile episode. This preemptive approach was fully applied in Period 2, and all the KPCKpBSI patients received initial active therapy and KPC-KpBSI-related mortality dropped to $6 \%$, as had already been observed applying the same strategy on a small population of HSCT carriers (9). The multivariate analysis confirmed that only the initial active antibiotic treatment was independently associated with survival and, differently from other reports (7-8), AML and shock were not predictors of mortality.

The role of initial active treatment in hematological patients with CR-KpBSI is critical. We previously reported the $90 \%$ of mortality in patients not receiving initial active treatment (8); another study observed inappropriate initial treatment in the $78 \%$ of non-survivors, associated with 21 -day mortality and significant predictor of mortality (7). In the present study the overall KPC-KpBSI mortality rate in KPCKpneumoniae carriers not receiving initial active treatment was $88 \%$, but reduced to $13 \%$ in those initially treated with at least one active antibiotic, and even to $9 \%$ with initial active combinations. The combination of active drugs had already been associated with a lower mortality rate (5) and independently associated with survival (7), and as a matter of fact in Period 2 all but one KPC-KpBSI patients received an active combination as initial treatment.

The $61 \%$ of KPC-KpBSI observed in Period 1 were breakthrough and the outcome was fatal in the $72 \%$ of cases. In Period 2, the preemptive administration of active antibiotics at the onset of febrile neutropenia could have prevented breakthrough KPC-KpBSI development, avoiding the overgrowth of the colonizing KPC-K.pneumoniae under inactive antibiotics and the subsequent blood-stream diffusion through the damaged mucosa, strongly contributing to the mortality reduction.

In high prevalence areas, active surveillance of the gastrointestinal tract is critical to avoid KPCK.pneumoniae nosocomial diffusion $(8-10,14)$, particularly in hematological wards where several conditions favor KPC-K.pneumoniae spread (8). In our experience we focused on the search and prompt detection of KPC-K.pneumoniae carriers, with the purpose of identifying the patients at the highest risk to develop KPC-KpBSI who would mostly benefit from the preemptive active strategy, obtaining a targeted initial treatment for KPC-KpBSI. In patients with hematological malignancies we strongly recommend the careful screening for KPC-K.pneumoniae rectal colonization, that represented the cornerstone of the preemptive strategy we customarily applied in Period 2: all KPC-KpBSI analyzed in the two study Periods developed in patients known as KPC-K.pneumoniae carriers but, in Period 2 all KPC-KpBSI were initially treated with active therapy. It is to be noted that in $25 \%$ of KPC-KpBSI developed in Period 2, the increased frequency of rectal screening avoided the delay in the active initial treatment due to unrecognized new carriers. 
Finally, the reduction in the probability of death from KPC-KpBSI led us to be less concerned about the occurrence of fatal KPC-KpBSI in prosecuting and scheduling intensive chemotherapeutic and/or HSCT programs in those patients identified as KPC-K.pneumoniae carriers.

Some considerations are needed. The preemptive strategy certainly increases the use of active drugs, and consequently a de-escalation of empirical active treatments should be carried out if KPC-K.pneumonia infection is not confirmed. Nephrotoxicity, especially in hematological patients receiving many other toxic drugs -chemotherapeutics, immunosuppressants, antifungals- may represent a limitation in colistin use (19); furthermore the emergence of resistance $(15,19-20)$ observed in $26 \%$ of our KPC-K.pneumonia blood isolates, makes colistin role uncertain. Ceftazidime-avibactam was widely used against KPCK.pneumonia infections with lower toxicity than colistin or aminoglycosides (19-24-25, 27); it proved active against all our KPC-K.pneumoniae blood isolates but resistance reported during (29) and independently (30) from previous ceftazidime-avibactam exposure, is troubling. New combinations with $\beta$-lactamase inhibitors, including carbapenem/ $\beta$-lactamase inhibitor combinations (26), could represent therapeutic alternatives also in the setting of hematological patients.

The monocentric design and the low number of patients included represent a limitation to the present report, and prospective larger studies are needed to confirm and generalize the results. Good-quality prospective randomized studies on CRE infections management are still needed, also in neutropenic haematological patients, and therefore our strategy -search of carriers and preemptive active treatmentobtained a noteworthy and promising reduction of KPC-KpBSI-related mortality in this population.

In conclusion, in high-risk haematological patients colonized with KPC-K.pneumoniae, the preemptive use of antibiotics active against KPC-K.pneumoniae as empiric treatment of febrile neutropenia resulted in a drop to $6 \%$ of KPC-KpBSI mortality rate, reduction of breakthrough KPC-KpBSI and probability of death for $\mathrm{KPC}-\mathrm{KpBSI}$, including AML patients.

\section{List Of Abbreviations}

KPC-KpBSI: KPC-K.pneumoniae bloodstream infection

CRE: carbapenem-resistant Enterobacteriaceae

HSCT: hematopoietic stem cell transplant

AML: acute myeloid leukaemia

\section{Declarations}

Ethics approval and consent to participate: The internal Ethical Committee of the Hematology, Sapienza University of Rome, approved this retrospective study.

Consent of publication: Not applicable 
Availability of data and materials: data supporting the results reported in the article can be found in archived database and are available from the corresponding author on reasonable request.

Competing interests: The authors declare that they have no competing interests

Funding: This research did not receive any specific grant from funding agencies in the public, commercial, or not-for-profit sectors.

\section{Authors' Contributions}

AM: conception and design of the study, acquisition of data, analysis and interpretation of data, and a major contributor in writing the manuscript.

GG: interpretation of data and a major contributor in writing the manuscript.

SS: performing and interpreting microbiological examinations

$\mathrm{CM}$ : acquisition and analysis of clinical and hematological data

SC: acquisition and analysis of clinical and hematological data

MLM: acquisition and analysis of clinical and hematological data

WB: contribution in the analysis of the patient clinical data

CC: contribution in the analysis of the patient clinical data

SMT: acquisition and analysis of clinical and hematological data

AMT: acquisition and analysis of clinical and hematological data

API: contribution in the analysis of the patient clinical data

GB: performing statistical analysis and contribution in reviewing the manuscript.

RF: contribution in reviewing the manuscript.

Acknowledgments: We thank Dr. Maura Di Pietrantonio for her thoughtful suggestions in reviewing the paper in English.

\section{References}

1. Satlin MJ, Jenkins SG, Walsh TJ The global challenge of carbapenem-resistant Enterobacteriaceae in transplant recipients and patients with hematologic malignancies. Clin Infect Dis 2014; 58(9) : $1274-83$ 
2. Pagano L, Caira M, Trecarichi EM, Spanu T, Di Blasi R, Sica S et al Carbapenemase-producing Klebsiella pneumoniae and hematologic malignancies. Emerg Infect Dis 2014; 20 : 1235-6.

3. Freire MP, Pierrotti LC, Filho HH, Ibrahim KY, Magri AS, Bonazzi PR C et al. Infections with Klebsiella pneumoniae carbapenemase (KPC)-producing Klebsiella pneumoniae in cancer patients. Eur J Clin Microbiol Infect Dis $2015 ; 34: 277-86$.

4. Girmenia C, Rossolini GM, Piciocchi A, Bertaina A Pisapia G Pastore D et al. for the Gruppo Italiano Trapianto Midollo Osseo (GITMO). Infections by carbapenem-resistant Klebsiella pneumonie in SCT recipients: a nationwide retrospective survey from Italy. Bone Marrow Transplant, $2015 ; 50(2)$ : 282-8

5. Tofas P, Skiada A, Angelopoulou M, Sipsas N, Pavlopoulou I, Tsaousi S et al. Carbapenemaseproducing Klebsiella pneumoniae bloodstream infections in neutropenic patients with haematological malignancies or aplastic anaemia: Analysis of 50 cases. Int $\mathrm{J}$ Antimicrob Agents 2016; 47(4) : 335-9.

6. Poucha SM, Satlin MJ. Carbapenem-resistant Enterobacteriaceae in special populations: solid organ transplant recipients, stem cell transplant recipients, and patients with hematologic malignancies. Virulence 2017; 8(4) : 391-402

7. Trecarichi EM, Pagano L, Martino B, Candoni A, Di Blasi R, Nadali G et al. for the Haematologic Malignancies Associated Bloodstream Infections Surveillance (HEMABIS) registry- Sorveglianza Epidemiologica Infezioni Funginein Emopatie Maligne(SEIFEM) group. Bloodstream infections caused by Klebsiella pneumoniae in onco-hematological patients: clinical impact of carbapenem resistance in a multicentre prospective survey. Am J Hematol 2016; 91 : 1076-1081

8. Micozzi A, Gentile G, Minotti C. Cartoni C, Capria S, Ballarò D et al. Carbapenem-resistant Klebsiella pneumoniae in high-risk haematological patients: factors favouring spread, risk factors and outcome of carbapenem resistant Klebsiella pneumoniae bacteremias. BMC Infect Dis 2017; 17: 203

9. Forcina A, Baldan R, Marasco V, Cichero P, Bondanza A, Noviello M et a.I Control of infectious mortality due to carbapenemase-producing Klebsiella pneumoniae in hematopoietic stem cell transplantation Bone Marrow Transplant, 2017; 52(1) : 114-119

10. Cattaneo C, Di Blasi R, Skert C, Candoni A, Martino B, Di Renzo N et al. Bloodstream infections in haematological cancer patients colonized by multidrug-resistant bacteria. Ann Hematol. 2018; 97(9) : 1717-1726

11. Zarkotou O, Pournaras S, Tselioti P, Dragoumanos V, Pitiriga V, Ranellou K et al. Predictors of mortality in patients with bloodstream infections caused by KPC-producing Klebsiella pneumoniae and impact of appropriate antimicrobial treatment. Clin Microbiol Infect, 2011; 17: 1798-803.

12. Bassetti M, Giacobbe DR, Giamarellou H, Viscoli C, Daikos GL, Dimopoulos G et al Critically III Patients Study Group of the European Society of Clinical Microbiology and Infectious Disease (ESCMID); Hellenic Society of Chemotherapy (HSC) and Società Italiana di Terapia Antinfettiva (SITA). Management of KPC-producing Klebsiella pneumoniae infections. Clin Microbiol Infect. 2018; 24(2) : 133-144. 
13. Giannella M, Trecarichi EM, De Rosa FG, Del Bono V, Bassetti M, Lewis RE et al. Risk factors for carbapenem-resistant Klebsiella pneumoniae bloodstream infection among rectal carriers: a prospective observational multicentre study. Clin Microbiol Infect 2014; 20 : 1357-62.

14. Amit S, Mishali H, Kotlovsky T, Schwaber MJ, Carmeli Y Bloodstream infections among carriers of carbapenem-resistant Klebsiella pneumoniae: etiology, incidence and predictors. Clin Microbiol Infect, 2015; 21 (1): 30-4

15. Conte V, Monaco M, Giani T, D'Ancona F, Moro ML, Arena F, et al. AR-ISS Study Group on Carbapenemase-Producing K. pneumoniae. Molecular epidemiology of KPC-producing Klebsiella pneumoniae from invasive infections in Italy: increasing diversity with predominance of the ST512 clade II sublineage. J Antimicrob Chemother. 2016; 71(12) : 3386-91

16. Freifeld AG, Bow EJ, Sepkowitz KA, Boeckh MJ, Ito JI Mullen CA et al. Clinical practice guideline for the use of antimicrobial agents in neutropenic patients with cancer: 2010 Update by the Infectious Diseases Society of America. Clin Infect Dis, 2011; 52 : 56-93.

17. Averbuch D, Orasch C, Cordonnier C, Livermore DM, Mikulska M, Viscoli C et al. on behalf of ECIL4, a joint venture of EBMT, EORTC, ICHS, ESGICH/ESCMID and ELN. European guidelines for empirical antibacterial therapy for febrile neutropenic patients in the era of growing resistance: summary of the 2011 4th European Conference on Infections in Leukemia. Haematologica, 2013 ; 98 :1826-35.

18. Bucaneve G, Micozzi A, Picardi M, Ballanti S, Cascavilla N, Salutari P et al. Results of a multicenter, controlled, randomized clinical trial evaluating the combination of piperacillin/tazobactam and tigecycline in high-risk hematologic patients with cancer with febrile neutropenia. J Clin Oncol, 2014; $32: 1463-71$.

19. Perez F, Chakhtoura NG, Papp-Wallace K, Wilson BM, Bonomo RA. Treatment options for infections caused by carbapenem-resistant Enterobacteriaceae: can we apply "precision medicine" to antimicrobial chemotherapy? Expert Opin Pharmacother 2016; 17(6) : 761-781.

20. Capone A, Giannella M, Fortini D, Giordano A, Meledandri M, Ballardini M et al. for the SEERBIO-GRAB network. High rate of colistin resistance among patients with carbapenem-resistant Klebsiella pneumoniae infection accounts for an excess of mortality. Clin Microbiol Infect, 2013; 19 : 23-30.

21. Tumbarello M, Viale P, Viscoli C, Trecarichi EM, Tumietto F, Marchese A et al. Predictors of mortality in bloodstream infections caused by Klebsiella pneumoniae carbapenemase-producing K. pneumoniae: importance of combination therapy. Clin Infect Dis 2012; 55 : 943-50.

22. Hirsch EB, Guo B, Chang K, Cao H, Ledesma KR, Singh M et al. Assessment of antimicrobial combinations for Klebsiella pneumoniae carbapenemase-producing K.pneumoniae. J Infect Dis, 2013; $207:$ 786-93

23. Daikos GL, Tsaousi S, Tzouvelekis LS, Anyfantis I, Psichogiou M, Argyropoulou A et al. Carbapenemase-producing Klebsiella pneumoniae bloodstream infections: lowering mortality by antibiotic combination schemes and the role of carbapenems. Antimicrob Ag Chemother, 2014; 58(4) : 2322-8. 
24. van Duin D, Lok JJ, Earley M, Cober E, Richter SS, Perez F et al. Colistin Versus CeftazidimeAvibactam in the Treatment of Infections Due to Carbapenem-Resistant Enterobacteriaceae Clin Infect Dis, 2018; 66: 163-171

25. Tumbarello M, Trecarichi EM, Corona A.De Rosa FG, Bassetti M, Mussini C et al. Efficacy of ceftazidime-avibactam salvage therapy in patients with infections caused by Klebsiella pneumoniae carbapenemase-producing K. pneumoniae. Clin Infect Dis 2019; 68 : 355-364

26. Zhanel GG, Lawrence CK, Adam H, Schweizer F, ZelenitskY S, Zhanel GG et al. Imipenem-Relebactam and Meropenem-Vaborbactam: two novel Carbapenem- $\beta$-Lactamase inhibitor combinations. Drugs 2018; 78(1) : 65-98

27. Castón JJ, Lacort-Peralta I, Martín-Dávila P, Loeches B, Tabares S, Temkin L et al. Clinical efficacy of ceftazidime/avibactam versus other active agents for the treatment of bacteremia due to carbapenemase-producing Enterobacteriaceae in hematologic patients. Int J Infect Dis, 2017; 59 : $118-123$

28. The European Committee on Antimicrobial Susceptibility Testing. Breakpoint tables for interpretation of MICs and zone diameters. Version 9.0, 2019. http://www.eucast.org/clinical_breakpoints/.

29. Spellberg B, Bonomo RA. Ceftazidime-Avibactam and Carbapenem-Resistant Enterobacteriaceae: "We're Gonna Need a Bigger Boat". Clin Infect Dis, 2016; 63(12) : 1619-1621

30. Gaibani P, Re MC, Campoli C, Viale PL, Ambretti S. Bloodstream infections caused by KPC producing Klebsiella pneumonia resistant to Ceftazidime/avibactam: epidemiology and genomic characterization. Clin Microbiol Infect. 2020; 26(4):516.e1-516 